\title{
INTEGRATIVE TREATMENT OF PERSONALITY DISORDER. PART I: PSYCHOTHERAPY
}

\author{
Mirjana Divac Jovanovic ${ }^{1}$ \& Dragan Svrakic ${ }^{2}$ \\ ${ }^{I}$ Department of Psychology, Faculty of Media and Communications, Belgrade, Serbia \\ ${ }^{2}$ Department of Psychiatry, Washington University School of Medicine in St Louis, St Louis, USA
}

received: 24.01.2017;

revised: 15.02.2017;

accepted: 22.02 .2017

\begin{abstract}
SUMMARY
In this paper, we outline the concept of integrative therapy of borderline personality, also referred to as fragmented personality, which we consider to be the core psychopathology underlying all clinical subtypes of personality disorder. Hence, the terms borderline personality, borderline disorder, fragmented personality, and personality disorder are used interchangeably, as synonyms. Our integrative approach combines pharmacotherapy and psychotherapy, each specifically tailored to accomplish a positive feedback modulation of their respective effects. We argue that pharmacotherapy and psychotherapy of personality disorder complement each other. Pharmacological control of disruptive affects clears the stage, in some cases builds the stage, for the psychotherapeutic process to take place. In turn, psychotherapy promotes integration of personality fragments into more cohesive structures of self and identity, ultimately establishing self-regulation of mood and anxiety. We introduce our original method of psychotherapy, called reconstructive interpersonal therapy (RIT). The RIT integrates humanistic-existential and psychodynamic paradigms, and is thereby designed to accomplish a deep reconstruction of core psychopathology within the setting of high structure. We review and comment the current literature on the strategies, goals, therapy process, priorities, and phases of psychotherapy of borderline disorders, and describe in detail the fundamental principles of RIT.
\end{abstract}

Key words: personality disorder - borderline personality - psychotherapy-integrative treatment-reconstructive interpersonal therapy

\section{REVISITING THE BORDERLINE CONCEPT}

We posit that all validly diagnosed personality disorder (PD) subtypes, such as narcissistic, histrionic, or dependent, among others, share the same core psychopathology referred to as "borderline level of functioning". The latter is characterized by intrapersonal indicators (fragmented and dysfunctional intrapsychic substructures manifested through primitive defenses, diffuse identity, partially impaired sense of reality, with largely intact reality testing, permissive morality, etc.), and characteristic interpersonal indicators, such as intense but unstable interpersonal relations, oscillating between idealization and devaluation, self-serving, and/or manipulative behavior with no empathy for others, yet marked by intense fear of being abandoned and left alone, etc. Frequently misdiagnosed as personality disorder are well adapted individuals with pronounced but normal traits (e.g., excessively shy or adventurous people), maladapted individuals with character disorder but no underlying fragmentation of personality (e.g., habitually violent, arrogant, or suspicious people), or individuals with neurotic character traits resulting from inflexible defense mechanisms (e.g., extreme stubbornness or scrupulousness). Differential diagnosis also includes affective disorders, especially treatment refractory depression and the so called Bipolar II disorder, and psychotic disorders because brief psychotic episodes are relatively common in patients with fragmented personality structure.
PD is an alloplastic disorder as these individuals initially attempt to change their environment (rather than their own behavior, or themselves) when faced with problems or stress. In contrast, individuals with anxiety disorders (formerly "neuroses") typically first seek to correct themselves and their behavior in an attempt to find a solution to an acute problem or stress (the so-called autoplastic disorders). Individuals with PD tend not to perceive their own immature characteristics and maladaptive behaviors as problematic, but, rather, as understandable, justified, and normal (the so-called ego-syntonic symptoms). The point is that maladaptive styles of various PD subtypes reflect a suboptimal, but purposeful solution that helps organize dissociated personality fragments into subtype-specific motivations, assumptions, and behaviors, thereby protecting the individual from the continual threat of disintegration. In therapy, the patient frequently has to abandon the advantages of the maladaptive solution in order to develop more authentic and less dictatorial structures of self and identity. Such dynamics has been dubbed U-shaped, evoking that "it has to get worse before it can get better". The three characteristics (alloplasticity, egosyntony, and the initial loss of stability, albeit maladaptive) provide the explanation as to why some persons very rarely seek help on their own, while the "U-shaped" dynamics explains why most people show resistance to change in general. 


\section{THE INTERACTIVE RELATIONSHIP BETWEEN PSYCHOTHERAPY AND PHARMACOTHERAPY}

Therapy of personality disorders most often combines pharmacotherapy and psychotherapy. Pharmacological control of disruptive symptoms (anxiety, depression, aggression) does not address the root cause of these negative moods, embedded in the fragmented personality structure. The benefits of pharmacotherapy are not limited to a mere cover-up of symptoms. With impulsive subtypes of personality disorder (narcissistic, histrionic), symptomatic treatments of anxiety and depression clear the stage for psychotherapy to promote integrative processes (i.e., more cohesive structures of the self and identity), ultimately establishing self-regulation of mood and behavior. A more complex dynamics is observed with causal pharmacotherapy of excessive Harm Avoidance (HA), a normal temperament trait which, if excessive, becomes the neurobiological risk factor for PD, also referred to as trait vulnerability for PD. Excessive HA (anxiety, fear) interferes with early integrations object relations, which remain partial. Partial object relations propagate through development and interfere with integration of ensuing structures (self, identity, character, morality). In the process excessive HA also shapes behavioral features of the individual, such as passive avoidance, cautiousness, fatigability, and continual "hardwired" anxiety, all typical of dependent, passive-aggressive and anankastic PD subtypes. In addition to having anxiety associated with HA, these individuals also have disintegration, separation, and existential anxiety, all functional anxieties associated with the fragmented personality core and precarious cohesion of the self. Here, pharmacological modification of hardwired trait vulnerability builds the stage for psychotherapy to promote integrative processes, eventually leading to bidirectional positive feedback between toned down neurobiology and more mature psychology. Pharmacological dulling of anxiety beyond the necessary comfort level is counterproductive as it may blur the cues the patient needs to develop important insights in psychotherapy.

Reconstructive psychotherapy is hard to implement in the setting of unstable affects, and/or risky or selfdestructive behaviors, all very common in the initial stages of treatment. Medications prescribed with expertise achieve a relatively prompt, visible, albeit nonspecific, improvement in the disturbing affects or risky behaviors. As a result, the patient begins to feel "much better" and pharmacotherapy thus sets a more suitable platform for the establishment of the psychotherapeutic relationship.

As hinted above, psychotherapy and pharmacotherapy have a bidirectional, modulating relationship: in addition to their independent effects, they also interact in a positive feedback manner. The common assumption that pharmacotherapy only works at the biological level and that psychotherapy only at the level of psychological processes is not exactly true. Psychotherapy can modulate structural CNS characteristics, such as receptor activity and expression in the brain through epigenetic mechanisms (Karlsson et al. 2010, Hirvonen et al. 2011). Paradoxically, after years of downplaying its efficacy, psychotherapy currently receives the strongest empirical support from basic neurosciences. On the other hand, pharmacological improvement of depressed mood or symptoms of psychosis can indirectly help restore complex psychological phenomena, such as identity cohesion, as shown in the example of pseudoborderline syndrome, characterized by transient identity confusion during an episode of Major Depression or Postpartum psychosis.

Targets for psychotherapy and pharmacotherapy are not completely interchangeable. Medication is more effective in stabilizing mood or modulating aggression and rather ineffective in changing internalized concepts about self and the world. Symptoms such as intensive but unstable interpersonal relationships or risky and self-destructive behaviors are clinically impressive but non-specific of PD. These symptoms represent a visible phenotypic expression of the underlying fragmented personality structure, most notably identity diffusion, precarious cohesion of the self, and lack of sublimatory channels. Modern pharmacotherapy has not yet accomplished a precise neural-molecular dissection of complex subjective mental phenomena to be able to influence them directly. Many of these phenomena are the so-called "emerging properties," i.e. features that unpredictably emerge from many-to-many interactions of lower order components, making biological dissection even more difficult. Psychotherapy remains the only approach - at least for now - to correcting the complex psychological processes and personality structure. It approaches personality structure top-to-bottom (i.e. from phenotype to epigenetics) and hence can correct complex phenomena (e.g. identity diffusion) without having to decipher their neural structure (if such structure indeed existed). The only direction pharmacotherapy can approach personality structure is bottom-totop (from molecular mechanisms to phenomenology). This direction requires knowledge of precise neurobiological mechanisms of subjective phenomena to be able to correct them specifically. Such highly specific pharmacology may well be an unattainable utopia. In our view, it would be unrealistic to expect that medicine of the future will be able to understand every detail of our human psyche based on blood tests, functional MRI or any other new method, no matter how sophisticated. However, for some psychiatric disorders, specific pharmacotherapy is an attainable ideal. Recent advances in our understanding of the phenotypic-genotypic architecture for eight clinical classes of the group of Schizophrenias indicate that a precise dissection of the molecular-modular mechanisms underlying this group of diseases is possible (Arnedo et al. 2015). 


\section{PSYCHOTHERAPY OF INDIVIDUALS WITH BORDERLINE DISORDER}

We shall first outline specific psychotherapeutic strategies, before proceeding with a detailed description of our approach to the psychotherapy of borderline disorders. As a general rule, psychotherapy of these individuals is complex and demanding. Due to the fragile, fragmented structure underlying $\mathrm{PD}$, psychotherapy is required to be, on the one hand, in-depth and reconstructive, and, on the other hand, highly structured, directive, and practical. Historically, these two essential requirements have been regarded as mutually exclusive. Also, psychotherapy is intensive, integrative, and transtheoretical by definition because it utilizes different types of discourse to match the multi-causality and multidimensionality of the disorder. The existing albeit relative consensus about the basic principles of the psychotherapeutic strategy for PD (Bateman 2004, Bateman \& Fonagy 2001, 2004, 2008, Akhtar 1995, Kernberg 1989, 2008, 2016, Tasman 2005) includes the following specific features with respect to:

a) therapeutic approach;

b) priorities in therapy;

c) the psychotherapeutic process;

d) goals of psychotherapy (from both the therapist's and the patient's perspective); and

e) the stages of therapeutic changes.

\section{Therapeutic approach}

The basic principles of the therapeutic approach to persons with BPD can be summed up using the following features:

- Psychotherapy is a long-term strategy and is concerned with all-inclusive patient-therapist relationships, not only interactions during the therapy sessions;

- Stability of the therapeutic framework and the highlystructured therapeutic process (order and rules);

- Defining the hierarchy of priorities;

- Clearly defining the roles and responsibilities of the patient and the therapist;

- Active role of the therapist;

- Establishing behavior control on the part of the patient;

- Setting and maintaining limits;

- Flexible approach (readiness to depart from the "rules");

- Combining therapeutic formats according to needs (individual, group, and partner).

\section{Priorities}

Continual assessment of risk, concerning the patient, the therapist, and the therapeutic process is fundamentally important. The priorities addressed in therapy include:

- Suicidal and homicidal behaviors;

- Behaviors threatening to disrupt the therapy;

- Serious acting out in therapy and outside;

- Dishonesty, lying or concealing important events;
- Trivializing the content of the sessions;

- Pervasive narcissistic resistance;

- Breaking the therapeutic contract.

Maintaining the hierarchy of these priorities involves carefully documenting risky behaviors, attentiveness to transference and countertransference problems, and ongoing consultation and supervision.

\section{Psychotherapeutic process}

The basic principles of the psychotherapeutic process include:

- Strong therapeutic alliance and setting up therapeutic contracts;

- Intense and active involvement on the part of the therapist;

- Tolerance of intense negative (and positive) transference by the therapist;

- Ongoing monitoring and utilization of countertransference in the therapy;

- Establishing relationship between the patient's behaviors and feelings in the present;

- Increasing the patient's awareness of his/her own emotional states and those of others and interpreting them;

- Blocking acting out and non-gratification of selfdestructive behaviors;

- Focus, clarification and interpretation in the "here and now;"

- Bridging: linking separated (split off) fragments and parts (of the self and objects);

- Matching the goals of therapy to the etiology of an individual.

\section{Therapeutic goals - the therapist's perspective}

- Creating a positive and supportive emotional setting (the patient uses the therapist's positive emotions because he/she does not have own);

- Help the patient develop their representations of self and others that are multidimensional, cohesive, and integrated (and consequently reduce misrepresentations and distortions in social interactions);

- Help the patient develop the capacity for self-reflection (developing self-observation, mentalization, rational and critical thinking, insightfulness);

- Protect the patient's extreme rejection sensitivity;

- Strong support for the development of the patient's tolerance of ambivalence in the perceptions of self and others (reflected in the reduced need for protection using primitive defense mechanism);

- Ego-consolidation by way of integrating representations of self and others.

\section{Therapeutic goals - the patient's perspective}

Therapeutic goals, the patient's perspective, refer to what is the patient expected to master in the course of the therapeutic process:

- To develop a meta-position and self-observation;

- To interpret one's own and others' feeling and motives; 
- To accept one's own negative feelings and oneself when not doing well;

- To analyze own positions in communications and their consequences in interpersonal relationships;

- To control and check for projections in transactions;

- To develop an awareness of the meanings he/she attaches to developments and events;

- To learn how to assume the position of others;

- To respect one's own and others' interests;

- To assume responsibility for one's own behavior, feelings, and decisions;

- To bridge the split off aspects of self, others, and reality;

- To accept and tolerate uncertainty and pain;

- To develop the capacity to cope with depression resulting from separation;

- To learn how to tolerate one's own fragmented self;

- To accept letting go instead of constantly fighting for control;

- To cultivate honest and direct communication in relationships;

- To experiment with different roles and ego states;

- To give up the grandiose validation of one's own and others' feelings, etc.

\section{Stages of psychotherapeutic changes}

Stages of psychotherapeutic changes in patients functioning at the borderline level:

- The first stage of therapy is usually marked with self-destructive behavior and acting out, depression, and/or severe disintegration anxiety. The patient's relationship with the therapist is based on idealization (the therapist is perceived as a powerful patron, supervisor and care-giver). The patient is very demanding and reacts angrily if the therapist does not comply with these roles. The patients is usually socially dysfunctional at this stage, frequently requiring "comforting" and support, even though they rush into transference and cause strong countertransference. A strong therapeutic alliance is required for this stage to succeed.

- The above described characteristics persist in the second stage of therapy, with a lessening of selfdestructive tendencies and the need for contact with the therapist outside the therapy. The patient's dependence on the therapist is expressed more explicitly, replacing implicit demands for attention and evidence that the therapists "cares". Initial signs of better social adaptation are possible even this early, although the patient may try not to show this in therapy in order to retain the "special" status as the person with exceptional needs.

- Acting out is largely eliminated in the third stage of therapy, but "acting in" continues in the sense of testing behaviors in therapy and outside. The patient shows growing confidence that the therapist cares about him/her, but this is accompanied by more frequent expressions of hostility in reaction to the limitations or disappointments within the therapeutic relationship. The process of mentalization progresses, making possible successful analyses of transference and countertransference events in therapy, as well as of interpersonal conflicts outside therapy (Akhtar 1995)

- At the fourth stage, the scope of experienced and expressed affects expands in the therapy and outside, as the patient shows improved performance in social roles, assumes greater responsibility, greater professional ambition, and begins to develop friendships. In this stage, it is important to work with a patient on the ways he/she could sabotage his/her developments and to integrate that insight into his/her expectations, in order to control regressions.

The duration of particular stages is different with each patient. It is important to notice that the stages are interwoven and progress in a nonlinear, canonic manner, with many back and forth steps along the way.

\section{RECONSTRUCTIVE INTERPERSONAL THERAPY (RIT)}

For a long time, therapists sought to develop an optimal modality to address specific requirements entailed in the work with persons with "low" personality organization or "poor" personality integration (Millon \& Davis 2000) or, as we call it, the borderline level of functioning (Kernberg et al. 1989). We posit that the therapy has to be reconstructive, that is, working on restructuring personality at the level of object relations (like psychoanalytic psychotherapy), and at the same time highly-structured to respond to the specific psychodynamics of PD (like cognitive-behavioral therapies). Neither of the two modalities in their original form could meet both of these fundamental requirements at the same time. The emergence of humanistic therapies, the systemic perspective, and the development of psychoanalysis in the direction of the relational paradigm, opened up space for a synthesis of the intrapsychic and interpersonal domains in the psychotherapeutic process. The emergence of existentialist and interpersonal therapies produced a variant of humanistic therapies that was eclectic and free to combine everything that was useful in achieving therapeutic change. The flexibility and eclecticism gave the new therapies the potential to integrate the two requirements in both the theoretical and practical sense (Dryden 1996). Reconstructive Interpersonal Therapy (RIT), as we implement it in practice, attempts to combine the intra-psychic and interpersonal discourse. By analysis of interpersonal relationships the RIT attempts to reconstruct internal personality structure (Divac Jovanović 1984, Divac Jovanović \& Lečić Toševski 2011). RIT is truly transtheoretical as it combines the in-depth psychodynamic, existentialist-humanistic and cognitive paradigms, all this to get as close as possible to an integrative, multifocal approach. The latter is the modality of choice in contemporary psychotherapy in general (Prochaska \& Norcross 2003). 
While fundamentally rooted in relational transactional analysis, RIT combines analytic methods (work with transference, utilization of countertransference, the concepts of object relations and mentalization) with elements of cognitive-behavioral techniques (strengthening the Ego state of the adult, decontamination of irrational beliefs, therapeutic contracts about behavior, etc.). In addition to applying psychotherapy in the strict sense of limits and rules, RIT emphasizes the importance of flexible strategy in psychotherapy of PD. This means that the therapy takes place not only in the sessions, but also in every other type of contact with the patient, including meeting patients in passing, if they are hospitalized, telephone calls, recorded messages, periods between sessions, etc. Since PD is pervasive (it affects many aspects of personality), the therapy has to be pervasive as well: every interaction with the patient is part of therapy. Hence, therapy has to be thoroughly thought through and planned, yet creative and flexible enough to adjust to every patient in every possible situation.

RIT is flexible with regard to technical neutrality, degree of control of the psychotherapeutic process, and the therapist-patient hierarchy, and is thus tolerated by PD patients with an excessive need for control and problem of staying in therapy (Joines \& Stewart 2002). The stages of the therapeutic process are similar to other therapies, yet specific as it requires explicit contracts that activate the patient's responsibility, i.e. the cognitive aspects of personality, at the very beginning of the treatment (similar to the postulates of existentialist psychotherapy). This places the patient in the role of a responsible participant who is required to master certain knowledge in the field of psychology, and thus makes the patient's participation in the treatment active and empowering. At the same time, similar to analytic treatments, RIT focuses on the therapeutic relationship, notably on unlimited „containing“ of all turbulent affective contents, tolerating projective mechanisms, bridging of the splitting mechanism, etc. The ultimate goal is to involve the patient in corrective emotional experience with the stable object who, in addition to confronting nonfunctional behavior, constantly ,grants permissions" to the patient to be, to exist, to feel and think. The therapy also involves working on the value system, which is typically insuficient with persons suffering from PD, using the narrative and the therapist's experience. RIT is guided by the rule that „everything that is not harmful is allowed“ and other rules are continually adapted to the individual patient during different stages of therapy. In addition to serving as ,a projection screen,“ the therapist is also a real human being involved in the patient's life and can show feelings, including explicit feelings that he/she cares about the patient (Novellino 1984), always a powerful message. Since RIT is based on empathy, this aspect of therapy involves exchanges of affection and acceptance, and, along with confrontation, comes quite close to a „natural“" relationship in interactions.
RIT's focus is first on the nurturance of the therapeutic relationship, and second on change. Once the patient feels that we understand and accept them, regardless of how „bad“ they were (the method of empathic transactions), they find it easier to talk about feelings and thereby to activate and develop their true self. As Akhtar (1995) stated, if the therapist misses the opportunity to express empathy, he/she can immediately see how hurtful this is to the patient. In such a situation the therapist must be prepared to retreat, and prevent harming the process, including asking the patient for advice about what he/she as therapist needs to do order to repair the injury. The therapist should make it perfectly clear that absolutely everything can be talked about in the session, especially if it involves topics critical to the therapeutic relationship and process. The patient should be helped to recognize the defensive function of idealization, and the therapist should observe his/her own positive transference and countertransference feelings and utilize them directly in the session to identify and possibly confront forms of primitive defense mechanisms. The basic goal is to help the patient see $\mathrm{him} /$ herself in a realistic light, and learn how to tolerate negative reactions, disappointment and failure. The therapist needs to be exceptionally patient with regard to the emotional attitude embedded in the basic message of these patients (,I hate you, don't leave me...") and to be ready to find a way to ,accept and love what he/she actually does not like." This is what transactionalists have in mind when they advise the therapist to always be able to see the „wounded child“ in the patient. As an aside, the best-selling book „I hate you, don't leave me“ (Kreisman \& Strauss 1991) drew attention of the public worldwide to the specific manifestations and complicated interpersonal relationships of these persons.

\section{RIT: Basic principles of psychotherapeutic work}

RIT is truly transtheoretical as it strives to integrate the humanistic, pychodynamic, and cognitive-behavioral paradigms into the overall strategy of psychotherapy of PD. RIT upholds all the basic principles of the component approaches but emerges as a qualitatively new approach not reducible to the original components. RIT follows eight basic principles in the therapeutic work with borderline patients. Of note, the therapeutic steps follow chronologically in sequence from 1 to 8 , but the manner in which they unfold resembles a canon: the beginning of each new step does not mean the end of the preceding one. In fact, the preceding steps remain active in all subsequent steps (Divac \& Švrakić 2016).

Tuning in to the "emotional wave-length" of the patient: genuine empathy, the position of „not knowing", close observation of verbal and non-verbal signs, "letting" the patient take control, unlimited "containing", the latter gives the patient the opportunity to deposit all unacceptable feelings and impulses, especially important for rage, onto the therapist. All this is done in order to win the patient over to engage and stay in. From the start, the therapist expects a negative 
transference and is prepared to monitor countertransference in order to be able to endure this demanding and exhausting relationship.

Keeping the patient informed and establishing the therapeutic and protective contracts. RIT follows the rule that the process and the logical foundation of treatment should be made known to the patient. In practice, this means that we inform the patient about the basic principles of psychotherapy (e.g., confidentiality, transparency, the need for patience, among others), about the logical foundation of therapy, and about what they can do to make progress. The therapeutic contracts incorporate a defined desired change and/or the patient's needs. The process of building the contract usually takes time, during which possible options crystalize, and the psychotherapeutic relationship builds. Protective contracts (antisuicide and antihomicide) are made to control acting out of any kind (which, if continued, would threaten the therapeutic process). Early experience of control over his/her behavior in therapy gives the patient an initial sense of success and reinforces positive motivation. Keeping the patient informed also involves providing simple explanations (mostly) of emotional states the patient directly asks about.

Analyzing the patient's ongoing relationships (including the therapeutic relationship) by monitoring the priorities in the contents brought to the session. Borderline patients "rush into" the transference with the therapist or engage in relationships outside the sessions that are laden with transference dynamics. The therapist absorbs the transference in order to preserve the therapeutic relationship. This stage can take long, and implies providing comfort and showing empathy for the patient's sense of injury or injustice until all the conflictual relationships of the patient are exhausted. Recognition of the repetitive patterns of interpersonal conflicts is then introduced very slowly.

Conditions for introducing primary objects into the analysis of transference relationships appear gradually and imply linking the feelings in the "here and now" with those in "there and then." This is a major step because it leads to the insight about one's unconscious motivation operating in ongoing relationships. Based on numerous conflicts with the therapist, superiors, husband/ wife, friends etc., the patient recognizes the repetitive behavior pattern and the attendant feelings (deconstructed in the analysis of "mind" games), on the one hand, and his/her unfulfilled genuine unconscious needs, on the other. At this stage, it is crucial for the patient to make progress, i.e., to recognize and adequately interpret own feelings, first in the present, and then in the past as well.

"Reframing" refers to a change in the frame of reference. In other words, reframing means giving an alternative meaning to the known content (setting it into a different frame, looking at it from different anggles). Reframing helps the work on accepting pain, loneliness, fear, and sorrow, which then leads to decontamination of grandiosity about feelings. This is a regressive stage which can last long and also occasionally return.
Analyzing options for fulfilling the newly uncovered needs but in ways other than self-injurious behavior, and practicing new behaviors with a strong support of the therapist (and the group). Here belongs the anticipation of possible self-sabotage and the expected possibility of returning to previous patterns, all this to preserve motivation should symptoms return (which happens frequently).

Nurturing the therapeutic relationship, notably corrective emotional experience and protection by way of ongoing verification of the interpersonal relationship.In this respect, the therapist's availability, generosity, and patience with repetitive symbiotic demands for support, approval, and contact are very important.

Open-end therapy: the therapy with borderline patients does not actually end. The therapist remains available, mostly for consultation, support, and coaching.

\section{Affective resonance: important diagnostic and therapeutic tool}

It is said that psychotherapy of persons with fragmented personality involves two patients, not one (Searls 1986). This is in reference to the specific nature of the bidirectional relationship established (transference and countertransference, in psychoanalytic terms) which is central in both the diagnostic and therapeutic sense. The bidirectional relationship is a critical source of information. Successful monitoring and analysis of feelings of both sides are crucially important for effective psychotherapy. The therapist monitors not only the spectrum of the patient's feelings, whether openly expressed or hidden, but does the same with his/her own "resonance" to those feelings. Within a complex entanglement of own feelings and those that emerge as a resonance to the patient's feelings, the therapist identifies the patterns of the patient's earlier object relationships, the acquired matrices of feelings, behaviors, and meanings the patient attaches to interpersonal relationships. The therapist uses his/her own feelings as data in the here-and-now and, if most of his/her blind spots have been eliminated, he/she is able to distinguish between his/her own feelings and those stirred up by the patient (Yalom 2011). Communicating one's own feelings is a powerful tool for clarification and confrontation in the therapy of borderline disorder. Paradoxically, this kind of open communication can be introduced earlier and more directly than is advisable in the therapy of persons functioning at a higher level. By learning to recognize the feelings they engender in the therapist, the patient learns the social skills and empathy, the lack of which led to their disturbed interpersonal relationships in the first place. RIT encourages the patient to ask directly and openly about thetherapist's feelings or thoughts if he/she may have any assumptions about them. These questions are answered equally directly and honestly for both poles of feelings, negative and positive. A direct and honest interchange of feelings and experiences is an amazing catalyst in therapy. Therapy of persons with PD absolutely has to avoid the impression of "magic, mystery and authority." 
Working with borderline patients stirs up a kaleidoscope of feelings in the therapist along with a continual sense of unease in the background. The patients' primitive defenses, especially splitting and projective identification, generate a spectrum of complex and chaotic transferences in the therapeutic situation. Inordinately intense and 'raw' affects expressed by the patient make the therapist feel like he/she is continually engaged in a life-and-death struggle, as vividly put by Kernberg and his colleagues (1989), which is something everyone working with these patients has experienced. The therapist frequently has the impression that he/she is walking on eggshells because these persons are extremely sensitive to the therapist's choice of words and the smallest nonverbal signs. Because of the intense atmosphere they generate, persons with PD do become "special" to their therapists, inspiring in them a surprising optimism about the success of therapy despite objectively poor prognostic signs. The feelings these patients usually manage to engender in the therapist include:

- guilt (the patients accuse the therapist of not taking proper care of them, not liking them, of being unprofessional, or being responsible for the deterioration in their condition, etc.);

- fantasies about rescuing the patient (perceiving the patient as really helpless, requiring the therapists to do things for him/her, overstepping professional boundaries by allowing the sessions to last longer, disclosing personal problems, failing to bill the patient, meeting in inadequate settings, etc.);

- anger and hatred (patients project negative and raw affects onto therapist); sense of helplessness and worthlessness (the patient devalues the therapist's efforts, the therapists feels that he/she may lack the skills, is incompetent, and consequently withdraws or becomes defensive);

- anxiety and dread (the therapist almost constantly expects that he/she will say something wrong and provoke an angry outburst from the patient, the therapist may fear that the patient will sue him/her for being unprofessional, etc.).

There are three ways in practice to diagnose the patient's authentic feelings or mechanisms by relying on our (the therapist's) feelings: 1). therapist experiences the feeling that the patient is not aware of feeling ("shifting" of feeling); 2) the therapist feels the feeling others have in the contact with the patient in the hereand-now ("affective resonance"); 3) the therapist feels the feelings the patient's parents felt in the there-andthen (countertransference). It is the therapist's responsibility to distinguish and separate those three and to use them timely in the course of therapy.

In contrast to patients functioning at the neurotic level, borderline patients stir up intense feelings in the therapist and challenge their ability cope to the extreme. The therapist is frequently left thinking about the patient, experiencing different feelings long after the session, and feels burdened in his/her free time. These experiences are more frequently negative (hostility, fear, confusion, helplessness, boredom, etc.), than positive (attraction, strong fantasies about rescuing or curing through unconditional acceptance and love). They fluctuate, much like the patient's affects, and can become extreme. For example, the patient may initially delight the therapists with deep insights, and then bitterly disappoint with unforeseen regression. These patients frequently generate in their therapist a strong self-critical compulsion to "do something" and "speed things up," which increases the danger of overstepping professional boundaries. One way to retain control despite such compulsions is that the therapist asks him/herself what is it that needs to be done that the parent failed to do.

RIT uses countertransference as a diagnostic and therapeutic tool. RIT works with the transference exclusively, like other therapies; in the here-and-now (Divac Jovanović \& Tatar Radojković 1987). It is important to allow the transference to develop and then help the patient to separate the past from the present or, in other words, "to learn how to remove the posters" of their primary objects from the faces of the people who are around in the present (Novellino \& Moiso 1990). Work in the "here-and-now" requires the contents from the past that appear in therapy to be revived as if happening in the present and to be processed transactionally until a corrective option is reached. The most potent technique in working with the transference in this type of therapy is the "empty chair technique". Here, the patient is facing his/her own ego states, or projections, while the therapist directs the patient towards the possibility of deepening insights, redefining early decisions and expand options. This "empty chair" technique uses projection to enable patient to finish "unfinished business" with various and many people in the surrounding and clarifies the patient's own contribution to the interpersonal conflicts, which are abundant. The "parent interview" technique enables the therapist to address the patient's Parent ego state and this may reveal hidden motives of the true parent in the past. The patient can then accept his/her parents and their motives in a new way or, if required, the therapist, as the patient's ally, may "defeat the powerful enemy". It should be noted that this technique is applied cautiously and only in later stages of therapy after progress has been achieved in overcoming splitting (as this might otherwise deepen the splitting).

Working with persons with PD requires the therapist to maintain both the intrapsychic and the interpersonal focus in order to be able to monitor his/her own countertransference reactions, as well as the patient's reactions and transference. All this requires that the therapist engage in self-analysis, or, in other words, the therapist has the responsibility to see that his/her own materials enter the therapeutic arena. To be able to understand the development of the transference-countertransference relationship is it essential to understand the phenomenon and the concept of projective identification, as it is lies at the core of this process (Divac \& Švrakić 2016). 


\section{Corrective emotional experience: the pathway to change}

In interpersonal therapies, a corrective emotional experience refers to the object constancy of the therapist and to unconditional granting permissions to the patient to exist, feel and think (to be). Permissions are antithetic to "prohibitions" the patient received in the formative period and later transformed into early non-functional decisions about him/herself and others and about life in general. The experience of object constancy is achieved in the therapeutic relationship that is maintained despite the patient's extreme behaviors and negative feelings during therapy. In the patient's past experience, these negative behaviors caused rejection. Of course, the rejection does not happen in therapy, even though the patient is constantly attempting to provoke it by uncounsciously playing mind games ("reject me," "dump me"). The patient's extremely risky behaviors are confronted and controlled by therapeutic contracts, but threatening rejection is never made in therapy because this would reinforce the early conclusion that "others can't be trusted" or that "one should never show one's feelings." This specially applies to sadistic feelings of these patients, which they fear the most, and which have to be first contained and then transformed into acceptable framework.

Deconstruction of the sequence of emotional developmental with persons with PD points to a number of characteristic patterns. Their development was stalled during the most sensitive period in the rapprochement sub-phase of the separation-individuation stage, when the mother, because of her own immaturity/disorder/ stress was not ready to allow the child to strive for autonomy without anxiety, emotional blackmail, rejection, etc. ("not good-enough mother"). This refers to children who were inadequately loved by their mothers who either "clung" to the child and were preoccupied with him/her (anxious mother) or oppositely, were grossly uncaring and aggressively rejecting (psychotic/depressive mother). In contrast, neurotic persons were frequently not loved "enough" or felt "unloved"/neglected/ frightened as children by parents who frequently suffered from some type of PD.

These observations are supported by research results showing that mothers of children who later developed PD were frequently emotionally unstable or latently, transitorily or openly psychotic, had an affective disorder, or were depressed (Maffei 1993). As such, they had an unconscious tendency to convey "double meaning" messages ("Go away, but don't leave me" or "You can leave but don't come back", etc.) which regularly generate surprise, confusion or disappointment in the child. For example, a child experiences a sudden and paradoxical rejection from an otherwise overly devoted mother, and somehow has to accept ('swallow') it immediately in order to not complicate any further the relationship on which he/she depends in reality. This may lead to sadism and/or mistrust and/or dependency as the main characteristics of the child's later relation- ships with objects. As adults, the patient does not move forward out of fear that he/she may lose the people they depend on. The patient also avoids becoming close with people out of fear that he/she may be "swallowed" by these same people. In other words, when they become active and attempt to move in a positive direction, they get in contact with their experience of own early abandonment, along with the fear of separation and depression. Since they experience this fear as something which they cannot overcome, they behave impulsively to avoid feeling something that they cannot resolve. This is why interpersonal relationship of persons with borderline disorder are characterized by the unconscious message addressed originally to the mother, and then also to all later objects: "I hate you, don't leave me" (Kreisman \& Strauss 1991).

Most persons with PD have experienced serious traumas in their childhood. Some authors even estimate that as many $60 \%$ of these individuals have experienced sexual, physical and/or verbal abuse in childhood (Zanarini et al. 1989). Physical or emotional abuse and/or neglect exposes the child early to strong negative affects or sensations that he/she is incapable of analyzing or processing. As a result, intense fear becomes fixed and hinders further development of mature personality structure, especially affecting the capacity for mentalization. The latter is the main deficit in the patient's mental functioning, causing numerous difficulties. We will use several examples to illustrate this. Details of the narratives have been changed to protect patient privacy.

Example 1: A 40-year old female patient, had parents who both had jobs involving field work and frequent absences, so that she never knew "when shall they be back." The mother is described as emotionally "cold" and very ambitious, the father as highly successful and hyperactive. As the only child, she was adored by both parents, but also continually left alone with a succession of various caretakers looking after her. Her key memory of childhood is waiting for hours for the parents to return home. She developed affective instability and hypersensitivity that later interfered with her choice of partners and attachment to them, making all relationships utterly ambivalent, constantly alternating between positive and negative phases (either extremely sentimental or furiously angry and persecutory). Professionally successful as an adult, she had problems with professional effectiveness due to excessive narcissistic hypersensitivity and time management (constantly being late, similarly to her parents, compulsively repeating her early trauma). We understood this passive-aggressive symptom as an attempt at overcoming (or control), as self-punishment and revenge at the same time.

Example 2: A 38-year old female patient, was the only child. She is professionally successful without a partner or sexual relationships, suffering from episodes of depression, anxiety attacks, hypochondriasis, somatization with stomach problems, profoundly dissatisfied, and with a sense of having been cheated on by life. 
Socially isolated because "nobody is worth enough" to deserve close relationship with her, but dreads loneliness. In therapy she reveals, but is not aware that her father has a paranoid disorder (untreated), and that her mother is emotionally distant and cold. We understood that, by immediate somatization or panic attacks she protected idealized images of both parents, fearing that she would be abandoned if she feels her real feelings. In therapy the main challenge was to get her to see reality.

Example 3: A 27-year old female patient was the third child in the family. She is professionally successful. Occasionally manifests eating disorder (bulimia) and cyclothymia (frequent and intense mood fluctuations with no apparent cause) and problems with partner relationships. Subjectively feels better when not in a relationship, but wants to have a family. Symptoms worsen when she is in a relationship as she becomes extremely demanding, anxious, angry, paranoid, uncertain, until the relationship ends. It is revealed in therapy that the mother was secretly treated for paranoid psychosis, and that the father, a medical doctor, was in denial of his wife's illness.

Example 4: A 35-year old female patient, has problems in maintaining professional position or relationship. She is depressed, suicidal, and occasionally promiscuous. After two years of therapy, reveals being physically abused in childhood by excessive and inadequate hygienic manipulation and beatings by her mother (the mother, a medical doctor, has an undiagnosed and untreated psychotic disorder; the father was emotionally withdrawn, reserved and cold). As an adult, her choice of partners was inadequate in that she "attached," without any affect, instantly and totally either to persecutor (antisocial persons) or to partners significantly below her social status, first rushing into and then fleeing out of relationships.

Some form of emotional or physical abuse, including neglect, in childhood, and the impact of not-goodenough mother (or father, we might add if we accept the concept that the most important task of the father in the early stages of the child's life is to support the mother) can easily be reconstructed in all four patients, who were all engaged in long-term psychotherapy.

The most important process in the first stage of therapy that the therapist "contain" the patient's suppressed anger (sadism), fear and emotional pain, and facilitate the expression and processing of these contents. The critical period in the development of trust lasts as long as it is needed for the patient to develop confidence that even if he/she shows what he/she actually feels there would still "be people who would not do what the mother did" and/or who would sustain all the attacks of panic, anger or sorrow. If the therapist can endure through this relatively difficult phase, full of negative transference and complicated countertransference, he/she can expect to see the early signs of trust in the constancy of the external object that "does not hurt." At this time, the patients usually begin to develop attachment to the therapist. Here, we stress the importance of establishing and developing this initial phase of the relationship with the patient as crucial for the later stages of therapy because it places the therapist in the position of the "third parent" (Berger 1980). This initial phase of therapy is what TA refers to as "reparenting" (Schiff 1975), or bringing up the child again. Object constancy (therapist) is the key to establishing adequate therapeutic relationship as he/she creates conditions for the patient's corrective emotional experience as a first step towards healing. This is the basis on which the therapeutic process can continue, gradually leading to a reconstruction of personality.

\section{The idiosyncratic frame of reference: working with meanings}

The manner of thinking and relating to one's own feelings is quite specific and unique in persons with PD. An inexperienced therapist might make a mistake of attempting to finish the patient's sentence and "insert" him/herself in the patient's train of thought because he/she thinks that he/she understood the patient. Since these patients often complain that others do not understand and/or accept them (because they do not understand and accept themselves, i.e., their own feelings) this eagerness on the part of the novice therapist may be motivated by the wish to show to the patient howhe/she is actively participating and does this with empathy. However, a contrary effect is usually produced, because these patients frequently imply idiosyncratic meanings to their narratives. Soon enough, the therapist realizes the conspicuous need to qualify every single statement. The rule that the therapist should follow is: "Listen, don't interrupt, and wait!" The important thing to always keep in mind is that these patients "are never as well as they may seem when they are doing well, and never as bad as they may seem when they are not doing well" (Maffei 1993). They may create an impression that they have no insight even when their insight is quite profound, and conversely, they may say things indicative of their early uncritical thinking even after years of therapy.

When they explicitly ask for explanations of certain issues (a sign that they are developing trust or, curiosity, at least) these patients are genuinely grateful for being given valid information. Similarly to persons with psychotic disorder, persons with PD are very good at making use of direct answers and information because of their tendency to think literally and in concrete terms. In contrast to them, neurotic patients are more likely to become alarmed or concerned by the provided information rather than utilize it to correct thinking or behavior. As a rule, when the patients become inquisitive (most frequently, when they begin to compare their own understanding to that of others), the therapist should take this as a signal to present his/her own frame of reference openly and directly, without hesitation. This draws the patient's attention and arouses his/her interest 
in discussion regardless of whether they agree or disagree with what was stated. The patient needs all kinds of information from all areas of life to make their deficient ego state of the Parent complete. Indeed, they frequently ask questions, especially if they feel free to do so and that there is a person they can ask and whom they can trust. Persons with PD react very well to fundamental discussions and are not satisfied when told that "things are the way they are" just because a book or a "theory" says so. They prefer being given concrete examples or evidence in support of basic rules of life. In order to accept anything new and incorporate it into their frame of reference, the information needs to be rich in meaning. Since fascination plays such an important part in attracting their interest, important information should be given in a way that makes an impression. To be effective, therapists need to make an impact and believable much like artists, and the therapist's energy level plays a significant part in the therapy of these patients.

\section{Reframing: the "royal" road in psychotherapy}

Positive connotation, i.e., giving support and granting permission to the patient to accept him/herself despite personal difficulties and interpersonal problems, in order to be able to make desired changes is the legacy of humanistic therapies. Changing the meaning, i.e., putting old contents into new frames, brings the patient, relatively early in therapy, into the position of fully accepting him/herself before making the desired changes. One of the most important humanistic postulates is that therapeutic change is possible only from the position of genuinely accepting one's self. At the time, this discoursere presented a major departure from the elitist understanding of what is "healthy" and "correct" and who (the therapist, of course) "holds the truth" about optimal functioning. The most important understanding is that people come into therapy with problems they cannot solve, and that we reveal those they can solve. By attaching new meanings to old contents and themes, the patient begins to see options (instead of dismissing the very existence of options) and recognizes own ability to choose alternative options and write a different life story. This paradigm shift freed the patient from feeling helpless and inadequate, and allowed therapists to move beyond the false superiority of their position.

Following the domination of psychoanalytic theories of psychopathology and the long-standing pathocentric psychotherapy of the first half of the $20^{\text {th }}$ century (the age of neuroses), positive connotation and client-oriented therapy became the dominant position of humanistic and systemic psychotherapies in the second half of the $20^{\text {th }}$ century. The transition from rigid ethical conservativism to religious and ethical pluralism facilitated the emergence of the narcissism and self-love (the age of personality disorder). Such trends threatened to bring the humanistic idea of self-acceptance to absurdity. In psychotherapy of neuroses, the patient initially erects defenses against guilt and is super-ethical, only to show lack of empathy close to the end of therapy, potentially opening the door for paradoxical immorality. The new generations of non-psychotic patients, i.e., those with PD, positive connotation has a precisely opposite effect: in the beginning, psychotherapy is centered around "immorality" (lack of empathy) while at the end, it shifts to guilt and personal responsibility. The patient can truly accept responsibility only after they experience being accepted themselves. If not used cautiously (but unconditionally and uncritically), positive connotation can cause the patient to stagnate in therapy: both the patient and the therapist may be deceived by the illusion that the patient is doing well and that the therapy is effective. As an important indicator of improvement in the psychopathology of self-love or self-blame, narcissistic personalities begin to realize that positive connotation applies to other people as well, instead to only themselves. In contrast, with neurotic personalities, the opposite is true: they need to learn that positive connotation applies to them, instead only to others.

\section{"Re-parenting": the therapist as the third parent}

Psychotherapy of persons with severe personality disorder sometimes involves applying techniques which use regression as method. Similarly to the therapy of persons with psychotic disorders, borderline personalities can benefit from the "re-parenting" method (Schiff 1975). A supportive therapeutic setting in which "permissions" are granted instead of "prohibitions", enables the person arrested in development to mature, to overcome fragmented contradictory representations of self and objects and their relationships, and to form whole, realistic representations. Along this path it occasionally becomes necessary to accept deeply regressive reactions so that the patient may understand their function (e.g., a borderline patient inconsolably sobbing in the arms of the therapist). Reparenting is a long-term process which requires persistence from the patient and a great deal of patience from the therapist, much like the work of the parent bringing up the child in real life. As already noted, this is why the therapist is sometimes described as the third parent (Berger 1980). Of course, it is not possible to overstate the degree of responsibility implicit in this position of the third parent, as well as the high level of integration it requires from the therapist.

In contrast to the therapeutic work with patients functioning at higher levels of personality organization where the development of symbiotic relationship is not advised, developing dependence in persons with severe personality disorder is a necessary first step in therapy. After progress has been achieved in therapy, the work focuses on overcoming (i.e., abandoning) this symbiotic dependence and the patient learns how to be independent without guilt or fear. It can be fascinating to observe how the therapist's simple, casual, positive comments about the person (the being) of the patient, for example, about his/ her inherent uniqueness, can have a healing effect on the patient who may be hearing this for the first time. In order to be effective, of course, the remark has to be genuine. 


\section{Couple therapy: "mediation at a distance"}

The therapy of persons with PD frequently combines different therapy formats, individual and group therapy, and various family members, such as a parent, a spouse, or significant partner, all of whom may join therapy in the later stages. Instead of doing family therapy (which these patients rarely accept) we employ "distance" family therapy, whereby a family member is being influenced indirectly, through the patient's progress. If a partner session does takes place at all, the work primarily involves mediation rather than therapy in the strict sense and the therapist frequently has to "interpret" the patient's feelings to the partner, and vice versa. The partner therapy can only truly begin after this mediated "introduction."

Partners of persons seeking psychotherapeutic help may sometimes refuse to get involved even after being told that their participation would be useful for the outcome. This usually happens when the uncooperative partners have some form of PD themselves, usually with passive-aggression or violence. Such partner contributes to or aggravates the problems of the patient, who paradoxically may be the single "identified" patient of the sick relationships. In this case, partner therapy becomes"distance therapy" because the therapist tries to influence the system (patient-partner) by way of correcting the behavior of the patient and thus indirectly changing the behavior of his/her partner. The source of pathological communication frequently resides with the absent partner with PD, making the person seeking help not only the "identified patient", but is also the "instrument" of change in his/her partner. Distance therapy is impressively functional in practice and we see this as resulting from the fact that the main channel (gateway) of communication for PD is not verbal, but behavioral. The planned correction of the present partner's behavior towards the absent partner indirectly achieves change in both partners' behavior and communication (and thus also in their feelings).

When partners attend therapy together, one as the identified patient, and the other as the partner assumed to be healthy, it turns out relatively often that both have some form of PD, usually complementary subtypes, antisocial and histrionic, paranoid and dependent, among others. In our practice, we have seen couples who epitomize the clinical hypothesis about complementary choices, the hypothesis that certain styles of adaptation are mutually attractive (e.g. antisocial and histrionic, paranoid and dependent, etc.). In such cases, partner relations can be so disrupted that they exclude any possibility of dialogue, which requires the therapist to engage in mediation, instead of partner therapy proper. This is especially true of persons with Cluster B $\mathrm{PD}$, i.e., the "dramatics," as they represent a mutually attractive choice. Mediation in this case is a method of choice, since regular psychotherapy sessions are quickly and easily disrupted. Transformative mediation is a process focused on understanding the needs and feelings of the other partner and improving communication. With the consent from both sides, the therapist serves as an explorer and interpreter of feelings, behavior and thinking of one or the other partner. Joint sessions, at which plans and simultaneous tasks for both sides are adopted, can be combined with individual sessions to work with the partners separately (the combination is balanced). As observed in our practice, when the partners are simultaneously involved in individual therapy with two different therapists, they are more likely to grow apart and definitely split up without improving mutual understanding (because of intensified splitting). Paradoxically and contrary to the rules, it is better in cases like these that the same therapist does individual sessions with both partners despite the fact that this requires additional effort from the therapist who the couples see as both the culprit and the arbiter.

In cases when partners'behavior styles reflect the "fearful" or "eccentric" clusters of PD, they frequently develop an intensely symbiotic relationship, which excludes other persons from the system. This makes the therapy a great deal more difficult, with resistance and ambivalence from both sides. The patient frequently places the therapist in the position of arbiter who is expected to choose sides, or else risk being devalued in the course of therapy. The therapist needs to balance interventions carefully and again play the part of the "interpreter". Interventions seeking to reveal feelings behind certain behaviors, which one of the partners sees as undermining, are very useful. For example, a submissive passive-aggressive partner realizes that behind a paranoid attack by his partner lies her feeling of intense fear of abandonment, not anger, which is what the submissive partner fears. After this type of interventions, partners frequently manage to overcome splitting and resistance and stay in therapy.

\section{CONCLUSION}

Despite the plethora of rules, guidelines, and directives in the literature, therapists working with individuals with immature (borderline, fragmented) personality structure still face many unanswered questions and dilemmas concerning psychotherapy of severe PD. The therapy is both challenging (because of intense transference and countertransference) and not (because it allows direct approach and improvisation). It is both long-term and not (because of frequent interruptions the actual time spent in therapy may not be long). Unparalleled by any other patient population is the inevitable work on the ethical value system of the patient, usually requiring the therapist to eventually reveal their own ethical principles as reference. Also, the intelligence of the patient, e.g. his/her willingness to think and make decisions, is critical for successful therapy. The therapy is either fun (for both parties) or it should not proceed at all. As much as the patient benefits from treatment, the therapist benefits as well. We can testify to the latter, because the decades of 
trying to understand and help individuals with severe personality fragmentation, commonly called PD, also helped us become more mature and in general wiser about priorities in life.

\section{Acknowledgements: None.}

\section{Conflict of interest: None to declare.}

\section{Contribution of individual authors:}

Both authors participated in literature searches and analyses, writing of the manuscript as well as on approval of the final version.

\section{References}

1. Akhtar S: Quest for answers. A primer of understanding and treating severe personality disorders. Jason Aronson. New Jersey, London, 1995.

2. Arnedo J, Šrakić D, Del Val C, et al.: Uncovering the Hidden Risk Architecture of the Schizophrenias: Confirmation in Three Independent Genome-Wide Association Studies. American Journal of Psychiatry 2015; 172:139-153.

3. Bateman A, Fonagy P: Treatment of borderline personality disorder with psychoanalytically-oriented partial hospitalization, an 18-month follow-up. American Journal of Psychiatry 2001; 158:36-42.

4. Bateman A, Fonagy P: Psychotherapy for borderline personality disorder: Mentalization based tretment. Oxford: Oxford University press, 2004.

5. Bateman A, Fonagy P: 8-year follow-up of patients treated for borderline personalit disorder: Mentalizationbased treatment versus treatment as usual. American Journal of Psychiatry 2008: 165:631-638.

6. Berger J: The Third Parent: New Directions in Group Psychotherapy, Nolit, Belgrade, 1980.

7. Divac Jovanovic M: Reconstructing the frame of reference - Transactional-analytic model of psychotherapy with borderline disorders, Psychiatry Today 1986; 2-3:291-303.

8. Divac Jovanović M, Tatar-Radojković S: Treating borderline phenomena across diagnostic categories. Transactional Analysis Journal 1987; 17:4-10.

9. Divac Jovanović M, Lečić-Toševski D: Integrative model of interpersonal psychotherapy of borderline disorder, Psychiatry Today 2011; 1:47-65.

10. Divac Jovanović M, Švrakić D: Various Faces of Borderline Personality, Clio, Beograd, 2016.

11. Dryden W. (Ed.): Developements in psychotherapy: Historical perspectives. SAGE Publication. Thousand Oaks. California, 1996
12. Hirvonen J, Hietala J, Kajander J, Markkula J, RasiHakala H, Salminen JK, Någren K, Aalto S, Karlsson H: Effects of antidepressant drug treatment and psychotherapy on striatal and thalamic dopamine D2/3 receptors in major depressive disorder studied with [11C]raclopride PET. Journal of Psychopharmacology 2011; 25:1329-1336.

13. Jalom DI: The Gift of Psychotherapy, Psihopolis institut, Novi Sad, 2011.

14. Joines $V \&$ Stewart I: Personality adaptation: A new guide to human understanding in psychotherapy and counselling. Life space Publ. Nottingham and Chapel Hill, 2002.

15. Karlsson H, Hirvonen J, Kajander J, Markkula J, RasiHakala H, Salminen JK, Någren K, Aalto S, Hietala J: Research letter: Psychotherapy increases brain serotonin 5-HT1A receptors in patients with major depressive disorder. Psychological Medicine 2010; 40:523-528.

16. Kernberg O, Selzer MA, Koenigsberg $H W$ et al.: Psychodynamic Psychotherapy of Borderline Patients. Basic Books. New York, 1989.

17. Kernberg OF, Yeomans FE, Clarkin JF: Transference Focused Psychotherapy: an Overview and update. Int. J. Psychoanal 2008; 89:6001-620.

18. Kernberg OF: What is Personality? Journal of Personality Disorders. Guilford Press 2016; 30:145-156.

19. Kreisman JJ, Strauss H: I Hate You - Don't Leave Me: Understanding the Borderline Personality, Morrow, William \& Co, New York, 1991.

20. Maffei C: Borderline Personality Disorder. Perspective of Diagnosis. Bollati Boringhieri. Torino, 1993.

21. Millon T, Davis R: Personality disorders in modern life. John Wiley \& Sons. New York, 2000.

22. Novellino M: Self-analysis of countertransference in integrative transactional analysis. Transactional Analysis Journal 1984; 14:63-75.

23. Novellino M, Moiso C: The psychodynamic approach to transactional analysis. Transactional Analysis Journal 1990; 20:187-192.

24. Prochaska JO \& Norcross JC: Systems of psychotherapy. A transtheoretical analysis. Thompson, Brooks/Cole. Stamford, CN, 2003.

25. Tasman A: Beyond the ICD and DSM: Diagnosis, Comorbidity, and the Therapeutic Alliance in Borderline Personality Disorder, Psychiatriki 2005; (Suppl):171.

26. Schiff J, Schiff A, Mellor $K$ et al.: Cathexis reader: Transactional Analysis Treatment of Psychosis, Harper \& Row. New York, 1975.

27. Searls HF: My work with borderline patients, Jason Aronson. New York, 1986.

28. Zanarini MC, Gunderson JG, Marino MF, Schwartz EO, Frankenburg FR: Childhood experiences of borderline patients. Comprehensive Psychiatry 1989; 30:18-25.

Correspondence:

Associate Professor Miriana Divac Iovanovic, MD, PhD

Department of Psychology, Faculty of Media and Communications

Karađorđeva 65, Belgrade, Serbia

E-mail:mirjana.divac@fmk.edu.rs 